\title{
Inguinal Sac Abscess Masquerading as a Strangulated Inguinal Hernia after Antrectomy and Proximal Duodenectomy for Perforated Peptic Duodenal Ulcer
}

\author{
Michael T. Li,, Bedabrata Sarkar, J.M. Kofi Abbensetts, and Chaitan K. Narsule ${ }^{1}$
}

\begin{abstract}
Background: Patients who undergo surgery for a perforated duodenal ulcer are at risk of developing an intraabdominal abscess. Even in this setting, the possibility of developing an inguinal hernia sac abscess is rare. Case Presentation: We report the case of a 66-year-old male who underwent an antrectomy and proximal duodenectomy, truncal vagotomy, Billroth II gastrojejunostomy, and a Witzel tube jejunostomy for a recurrent perforated duodenal ulcer. Seven days after this operation, he developed symptoms concerning for a strangulated left inguinal hernia. At operation, a hernia sac abscess of Candida glabrata was identified and excision of the hernia sac and McVay herniorrhaphy were performed.

Conclusion: An inguinal sac abscess may develop in patients who have undergone surgery for perforated peptic ulcers and can masquerade as a strangulated inguinal hernia. In the post-operative period, it should be considered in patients who develop an acute onset of groin pain. Definitive management should include excision of the sac and herniorrhaphy without a mesh prosthesis to limit the risk of a chronic wound infection.
\end{abstract}

Keywords: inguinal sac abscess; peritonitis; perforated duodenal ulcer; McVay herniorrhaphy

A LTHOUGH THE USE of proton pump inhibitors, avoidance of non-steroidal anti-inflammatory drugs (NSAIDs), and treatment of coincident Helicobacter pylori infections have been effective in reducing the incidence of complicated peptic ulcer disease, perforated peptic ulcers of the stomach and duodenum do occur and result in peritonitis and contamination of the peritoneal cavity. After surgical management, abscesses can develop within the recesses of the peritoneal cavity in a delayed fashion. Even in this setting, an abscess presenting in an inguinal hernia sac is a rare event and can masquerade as a strangulated inguinal hernia. This entity was first reported in 1959 [1]; however, few additional reports have followed. This is a case report of an inguinal sac abscess after surgical management for a perforated peptic duodenal ulcer.

\section{Case Presentation}

A 66-year-old male with a history of peptic ulcer disease presented with acute onset abdominal pain. Notably, he had undergone a Graham patch omentopexy for a perforated proximal duodenal ulcer four years prior to presentation. On admission, the patient had peritonitis, and a computed tomographic (CT) scan of the abdomen demonstrated a perforation of the right wall of the proximal duodenum with extravasation of contrast (Fig. 1). At laparotomy, the perforation of the proximal duodenum was identified (Fig. 2) along with purulent peritonitis. Because the patient had a history of non-compliance with proton pump inhibitors since the prior Graham patch omentopexy, the patient underwent an antrectomy, proximal duodenectomy, truncal vagotomy, Billroth II gastrojejunostomy, and a Witzel tube jejunostomy.

On post-operative day seven, the patient developed an acute onset of pain and swelling of his left groin that was concerning for a strangulated left inguinal hernia. His initial leukocytosis had resolved and abdominal examination did not reveal peritoneal signs. At operation, however, the left inguinal canal was explored and an indirect hernia sac abscess was identified (Fig. 3). The abscess was drained, the

\footnotetext{
${ }^{1}$ Department of Surgery, Boston University School of Medicine, Boston, Massachusetts.

${ }^{2}$ Aventura Hospital and Medical Center, Aventura, Florida.
}

(C) Michael T. Li et al. 2017; Published by Mary Ann Liebert, Inc. This Open Access article is distributed under the terms of the Creative Commons License (http://creativecommons.org/licenses/by/4.0), which permits unrestricted use, distribution, and reproduction in any medium, provided the original work is properly credited. 


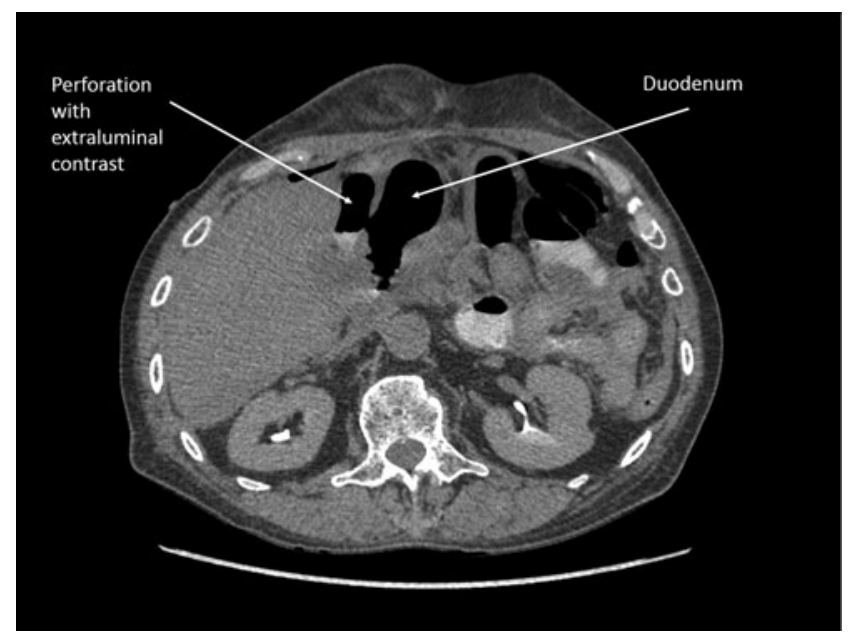

FIG. 1. Abdominal computed tomographic scan demonstrating perforation of the proximal duodenum with extravasation of contrast.
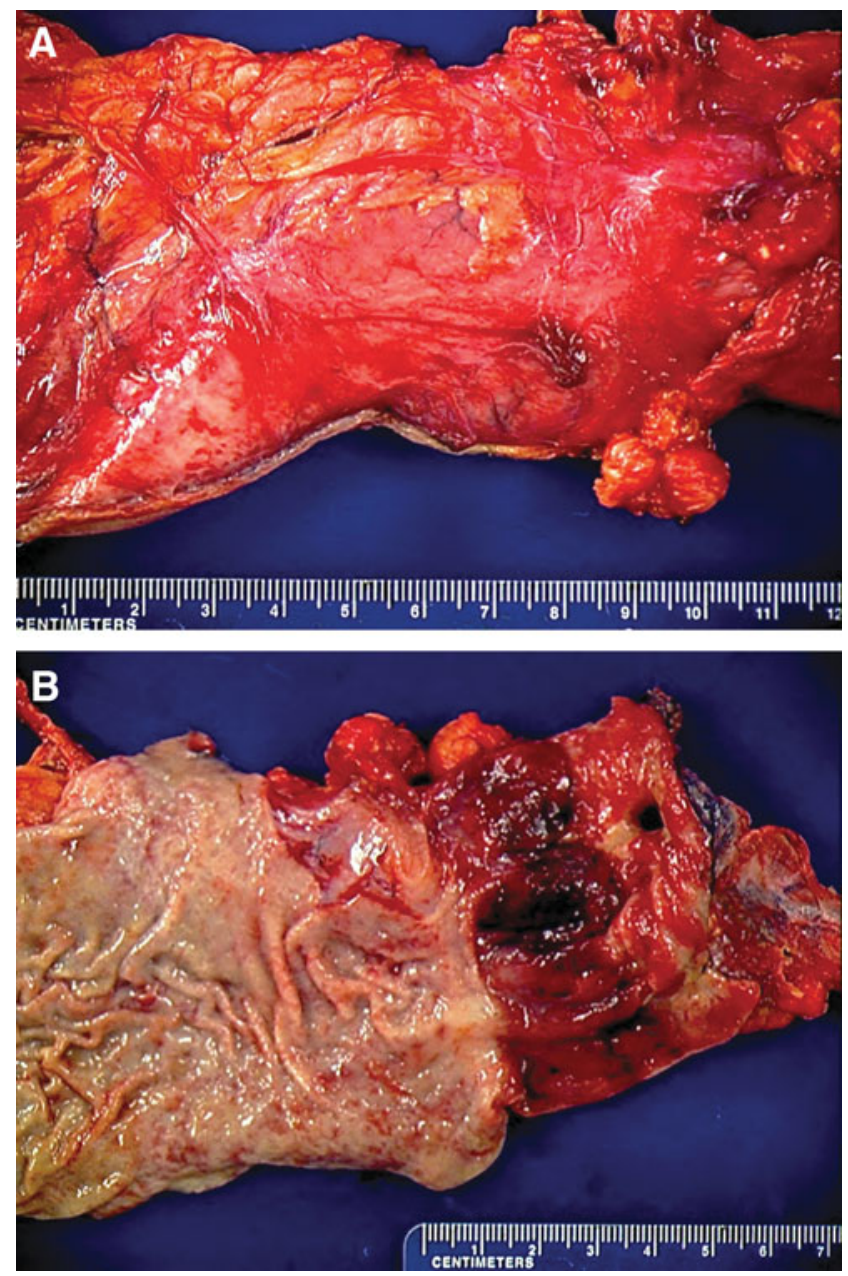

FIG. 2. (A) Antrum and proximal duodenum, resected, with perforated duodenal ulcer. (B) Surgical specimen, opened.

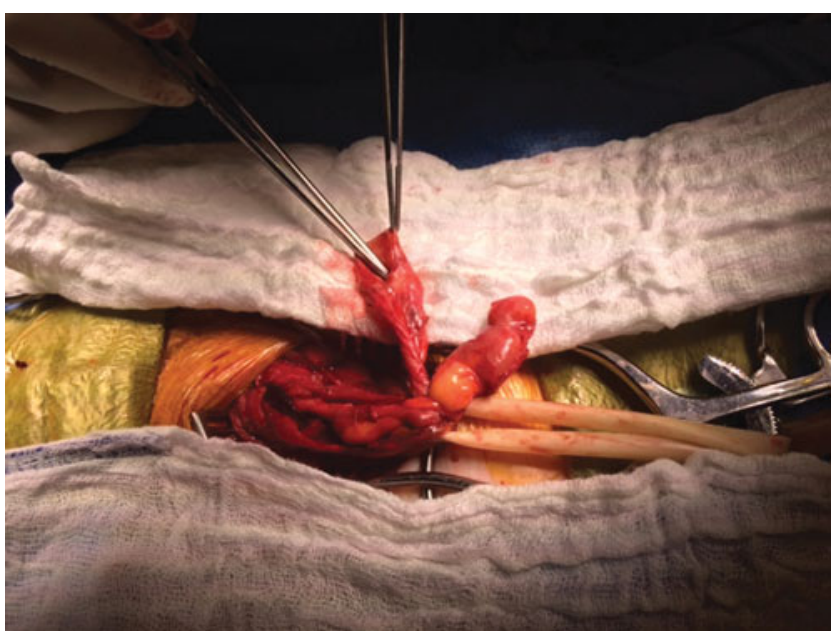

FIG. 3. Skeletonization of the inguinal hernia sac abscess during left groin exploration. (Orientation: Patient's legs are beyond the top of the picture, the upper body is beyond the bottom of the picture, left is patient's left, right is patient's right, and the Penrose drain is around spermatic cord structures.) The hernia sac is held by forceps, and a cord lipoma is present.

hernia sac was excised, and a McVay herniorrhaphy was performed. Cultures from the abscess grew Candida glabrata. The patient was treated with antifungal therapy and recovered without event.

\section{Discussion}

When considered separately, peritonitis (caused by perforation of the alimentary tract) and inguinal hernias are diagnoses that are neither rare nor infrequent in their incidence. However, the formation of an inguinal sac abscess resulting from peritonitis caused by perforation of the alimentary tract is a rare clinical entity. We report the case of a patient who developed a left inguinal hernia sac abscess after surgical management of a recurrent perforated peptic duodenal ulcer.

Perforated peptic ulcers with purulent peritonitis can predispose patients to the development of an intra-abdominal abscess. In this patient, who previously underwent a Graham patch omentopexy for a perforated duodenal peptic ulcer, it is believed that the patient's non-compliance with proton pump inhibitors caused a recurrence of the duodenal ulcer because he had no evidence of $H$. pylori infection on laboratory testing or other pathology. Because the patient had reported no symptoms or history of inguinal hernias, the development of left inguinal pain and swelling in the post-operative period was unexpected, especially with features that were concerning for a strangulated hernia. However, once the inguinal sac abscess was identified, drained, and excised, the microbiologic analysis confirmed that the perforated duodenal ulcer was the cause of the abscess. Ultimately, effluent from the perforated ulcer accumulated in dependent areas of the peritoneal cavity, which in this case included an unknown, sub-clinical indirect hernia sac that allowed for abscess formation.

After the initial reports of inguinal hernia sac abscesses following management of peritonitis by Cronin and Ellis in 1959 [1] and by Chary in 1977 [2], few additional cases have 
been reported. Other cases of hernia sac abscesses have been related to appendicitis, especially in the setting of Amyand or De Gargenot hernias [3-7], diverticular abscesses [8-10], Littre hernia [11], cancer [12], other colonic pathology [13], and even colorectal surgery [14]. Also, in an earlier era, several reports described cases in which a hernia sac abscess was identified before a diagnosis of generalized peritonitis was made [15-18], but this phenomenon may have been a result of the limitations of the diagnostic imaging modalities that were available at the time, and is not expected to persist in the modern era in which CT imaging enables surgeons to diagnose specific causes of peritonitis promptly.

Percutaneous drainage of a hernia sac abscess has been reported previously [13]. Yet, in the case presented here, surgical exploration was performed in part because a strangulated inguinal hernia was suspected. This approach would allow for effective treatment of both the abscess and inguinal hernia. Hernia sac drainage and excision with McVay herniorrhaphy was deemed to be safe in a contaminated environment and, after more than two years of clinical follow up, there has been no evidence of a hernia recurrence or other complications. It seems self-evident that herniorrhaphy with prosthetic mesh should be avoided in situations involving peritonitis and deep space infection. Furthermore, even if the existence of the inguinal hernia had been known at the time of laparotomy, surgical intervention for the hernia would have been deferred until the patient had recovered fully from the laparotomy for peritonitis to limit the risk of a surgical site infection in the inguinal wound and allow for a more durable mesh herniorrhaphy in the future.

\section{Conclusion}

An inguinal hernia sac abscess can develop after the operative management of a recurrent perforated peptic duodenal ulcer and may masquerade as a strangulated inguinal hernia. Surgeons should consider this entity in any patient who recently underwent a laparotomy for generalized peritonitis caused by a perforated hollow viscus and has acute onset groin pain. Although inguinal hernia sac abscesses can be managed percutaneously, any plan for sac excision and herniorrhaphy should avoid the use of a prosthetic mesh to limit the risk of a prolonged surgical site infection.

\section{Author Disclosure Statement}

No competing financial interests exist.

\section{References}

1. Cronin K, Ellis H. Pus collections in hernia sacs: An unusual complication of generalized peritonitis. $\mathrm{Br}$ J Surg 1959;46:364-367.

2. Chary S. Delayed abscess of hernia sac secondary to peritonitis. J R Coll Surg Edinb 1977;22:282-284.

3. Salemis NS, Nisotakis K, Nazos K, et al. Perforated appendix and periappendicular abscess within an inguinal hernia. Hernia 2006;10:528-530.

4. Kueper MA, Kirschniak A, Ladurner R, et al. Incarcerated recurrent inguinal hernia with covered and perforated appendicitis and periappendicular abscess: Case report and review of the literature. Hernia 2007;11:189-191.

5. Meinke AK. Review article: Appendicitis in groin hernias. J Gastrointest Surg 2007;11:1368-1372.
6. Gillion JF, Bornet G, Hamrouni A, et al. Amyand and de Garengeot hernias. Hernia 2007;11:289-290.

7. Sharma H, Jha PK, Shekhawat NS, et al. De Garengeot hernia: An analysis of our experience. Hernia 2007;11:235-238.

8. Andrabi SI, Pitale A, El-Hakeem AA. Diverticular abscess presenting as a strangulated inguinal hernia: Case report and review of the literature. Ulster Med J 2007;76:107108.

9. Greenberg J, Arnell TD. Diverticular abscess presenting as an incarcerated inguinal hernia. Am Surg 2005;71:208-209.

10. Tufnell ML, Abraham-Igwe C. A perforated diverticulum of the sigmoid colon found within a strangulated inguinal hernia. Hernia 2008;12:421-423.

11. Al Mogrampi S, Krexi A, Verroiotou M, et al. Inguinal abscess in a strangulated Littre's hernia. Surg Infect Case Rep 2016;1:1.

12. Boormans JL, Hesp WL, Teune TM, Plaisier PW. Carcinoma of the sigmoid presenting as a right inguinal hernia. Hernia 2006;10:93-96.

13. Mizuno R, Okuno M, Kojima H, et al. An abscess mimicking a left incarcerated groin hernia. Hernia 2012;16: 723-725.

14. Ikeda S, Takeda H, Yoshimitsu M, et al. Abscess in the inguinal hernial sac after peritonitis surgery: A case report. World J Gastroenterol 2009;15:1007-1009.

15. Sellu DP. Pus in groin hernial sacs: A complication of hernial sacs: a complication if non-generalised peritonitis. Br J Clin Pract 1987;41:759-760.

16. Cameron BH. Two cases of primary bacterial peritonitis presenting with a tender hernia. Aust N Z Surg 1991;61: 794-796.

17. Courtney ED, Melville D, Leicster RJ. Strangulated hernias secondary to generalized peritonitis: An old lesson relearnt. Hosp Med 2003;64:430-431.

18. Ekwueme O. Strangulated external hernia associated with generalized peritonitis. Br J Surg 1973;60:929-933.

Address correspondence to:

Dr. Chaitan K. Narsule

Division of Trauma and Acute Care Surgery

Department of Surgery

Boston University School of Medicine

One Boston Medical Center Place

Dowling 2 South

Boston, MA 02118

E-mail: chaitan.narsule@bmc.org

\begin{tabular}{|c|}
\hline Abbreviations Used \\
$\mathrm{CT}=$ computed tomography \\
NSAIDs $=$ non-steroidal anti-inflammatory drugs
\end{tabular}

Cite this article as: Li MT, Sarkar B, Abbensetts JMK, Narsule CK (2017) Inguinal sac abscess masquerading as a strangulated inguinal hernia following antrectomy and proximal duodenectomy for perforated peptic duodenal ulcer: Case report. Surgical Infections Case Reports 2:1, 58-60, DOI: 10.1089/crsi.2017.0011 